UDC 517.9

\title{
THE FIXED-POINT PROPERTY UNDER INDUCED INTERVAL MAPS OF CONTINUA
}

\section{ВЛАСТИВІСТЬ ІСНУВАННЯ НЕРУХОМОЇ ТОЧКИ ДЛЯ ІНДУКОВАНИХ ВІДОБРАЖЕНЬ ВІДРІЗКА НА КОНТИНУУМАХ}

\section{Robatian}

\author{
Inst. Math. Nat. Acad. Sci. Ukraine \\ Tereshchenkivska st., 3, Kyiv, 01601, Ukraine
}

\begin{abstract}
Let $f: I \rightarrow$ I be a continuous map of a compact interval I and $C(I)$ be the hyperspace of all compact subintervals of I equipped with the Hausdorff metric. We investigate the existence of the fixed-point property of subsets of $C(I)$ with respect to any induced interval map $\mathcal{F}: C(I) \rightarrow C(I)$. In particular, we prove that any nonempty subcontinuum of $C(I)$ has the fixed-point property.

Нехай $f: I \rightarrow I-$ неперервне відображення компактного інтервалу I та $C(I)$ - гіперпростір усіх компактних підінтервалів I з метрикою Гаусдорфа. Вивчається властивість існування нерухомої точки в підмножинах $C(I)$ відносно індукованого відображення $\mathcal{F}: C(I) \rightarrow C(I)$. Зокрема, доведено, щз будь-який непорожній підконтинуум $C(I)$ має властивість існування нерухомої точки.
\end{abstract}

1. Introduction. Studying the fixed-point property for different classes of mathematical objects has been attracting mathematicians' attention since decades ago. Particularly, over the past halfcentury the theory of fixed points revealed that it is an incredibly powerful tool in the study of various phenomena, as the fundamental concepts of "stability" or "equilibrium" might be described in terms of fixed points. That is why fixed-point techniques and theorems have been frequently applied in such diverse fields of science ranging from biology, chemistry, economics, engineering and physics to fields such as game theory and logic. In mathematics, and particularly in topology, the problem of the "fixed-point property" (or briefly FPP) is of great importance as well. A topological space $X$ is said to have the fixed-point property if for any continuous map $f: X \rightarrow X$ there exists $x \in X$ such that $f(x)=x$. Among topological spaces, Euclidean spaces were the first, for which the existence of the FPP was proved. This was done, more than a century ago, in theorems which are known as Brouwer's fixed-point theorems. In the most general form, the Brouwer's fixed-point theorems state that if $A$ is a compact convex subset of a topological space $X$, then every continuous map from $A$ into itself has a fixed point. Therefore, the simplest forms of Brouwer's fixed-point theorems guarantee the existence of at least one fixed-point in a compact interval or disk under any continuous self-map of that interval or disk.

In this paper, we study the FPP in the hyperspace of all compact subintervals of a compact interval $I$ with respect to induced interval maps on it. Before moving further, let us introduce the space we are going to consider in a more precise way.

Let $f: X \rightarrow X$ be a continuous map from a continuum $X$ into itself. Denote by $C(X)$ the hyperspace of all compact connected subsets of $X$ endowed with the Hausdorff metric. It is known that if $X$ is compact, so is the hyperspace $C(X)$ (see [9, p. 52-63]). We define the

(c) D. Robatian, 2015 
induced continuous map $\mathcal{F}: C(X) \rightarrow C(X)$ by setting $\mathcal{F}\left(x_{c}\right)=\left\{f(x): x \in x_{c}\right\}$ for each $x_{c} \in C(X)$. It is also known that continuity of $f$ implies continuity of $\mathcal{F}$. In the present work, we consider the particular case where the continuum $X$ equals the compact interval $I=[0,1]$. More precisely, we assume that $f$ is a continuous self-map of the interval $I=[0,1]$ and $C(I)$ is the hyperspace of all compact subintervals of $I$. Consequently, the induced map $\mathcal{F}: C(I) \rightarrow$ $\rightarrow C(I)$ will be a map such that $\mathcal{F}([a, b])=\{f(x): x \in[a, b]\}$, for any $a, b \in I$. It was observed that if $X$ is a one-dimensional continuum, then there could exist a close connection between the dynamics of the induced map $\mathcal{F}: C(I) \rightarrow C(I)$ and those of the original inducing map $f: C(I) \rightarrow C(I)$ (see $[3,4,6,8])$. In particular, it was proved that if we consider the induced map of the interval $I$, then the $\omega$-limit set of a point of $C(I)$ is either a union of singletons or a finite subset of $C(I)$. Nevertheless, the connection between the dynamics of an interval map $f$ and the induced map $\mathcal{F}$ on $C(I)$ is not always that close. For example, the induced map never happens to be transitive, even if $f$ is transitive [1] (more generally, an induced map of graphs is never transitive [7]).

The main question of this paper is then which characteristics a subset of $C(I)$ must have, so that the subset has the FPP. In order to do this, we will begin from the least complicated sets and will develop the discussion to some of those subsets which, roughly speaking, have more complex structures. Naturally, we will focus on the topological characteristics of these sets, since the FPP is, in fact, a topological property. That is, two homeomorphic spaces either both have or both lack the FPP (see [10]). Therefore, in Subsection 3.2, finite sets of $C(I)$ are going to be considered first, as they are apparently the simplest subsets of the space $C(I)$. In there, we will show that, in general case, these sets do not have the FPP. But it might also be observed that if one adds a minimum number of certain conditions to these finite sets, then there occurs a different story and the FPP holds for them. After that, in Subsection 3.3 we study a certain class of infinite subsets of $C(I)$ and prove that they do not necessarily include a fixed point. Although, they always have a point of period two. Finally, in Subsection 3.4 we prove that any nonempty subcontinuum of $C(I)$ has the FPP. Before moving onto the main part of the paper, we give some important definitions and explanations that are going to be used in the remainder of the paper.

2. Preliminaries. In the remainder of the paper we always assume that

$I$ equals the compact interval $[0,1]$ endowed with the usual Euclidean metric;

$C(I)$ denotes the hyperspace of all compact connected subsets of $I$, endowed with the Hausdorff metric;

the lowercase $f$ denotes a continuous interval map from $I$ into itself;

the uppercase $\mathcal{F}$ denotes the induced map of $f$ from $C(I)$ into itself, i.e., for any $a, b \in I$ we have that $\mathcal{F}([a, b])=\{f(x): x \in[a, b]\}$.

We would like the reader to remember that, for the sake of saving time and space, in most of the cases, especially when we state a new lemma or theorem, we introduce $\mathcal{F}$ without repeatedly mentioning that $\mathcal{F}$ is the induced map of $f$. So, whenever we talk about $\mathcal{F}$, we assume the existence of $f$ a priori.

In the forthcoming paragraphs, we would like to make some clarifying points regarding the phase space $C(I)$, as it helps us express the further argumentation more effectively on the one hand and more accessible for the reader on the other.

Firstly, note that we treat each element of $C(I)$, despite the set-nature they have, as a single whole object. That is, if roughly speaking, any space consists of points, then each point of $C(I)$ 


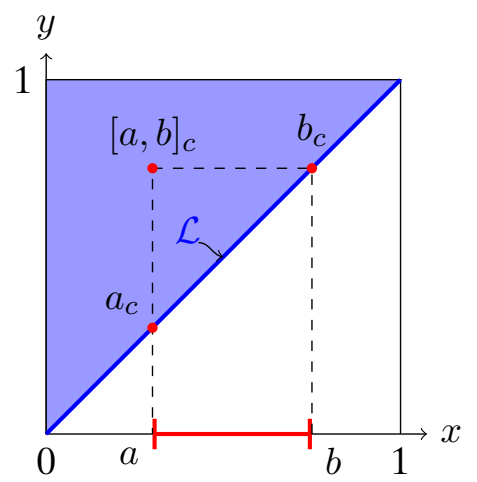

Fig. 1. $I=[0,1]$ is plotted on the horizontal axis, the shaded triangle shows $I_{y \geq x}^{2}$ and the dark black diagonal denoted by $\mathcal{L}$ shows the subset of all degenerate pointervals of $C(I)$. Points $a_{c},[a, b]_{c}, b_{c}$ in $I_{y \geq x}^{2}$ are related to $a,[a, b], b$ in $I$, respectively.

is a nonempty closed interval in $I$. For this reason and in order to avoid any ambiguity, we use the word pointerval to address an individual element of the space $C(I)$. Moreover, it is necessary to introduce an appropriate notation in order to remove any probable confusion. Let $a$ and $b$ be two arbitrary points in $I$ such that $a \leq b$, then the notation $J=[a, b]$ could potentially lead to confusion, since it is not entirely clear whether we mean the ordinary interval $[a, b]=\{x \in I \mid a \leq x \leq b\} \subseteq I$ or we mean the pointerval $[a, b]$ as a single element of $C(I)$. Therefore, to avert this misunderstanding, we use $[a, b]_{c}$ (or equivalently $J_{c}$ ) for a pointerval in $C(I)$ (i.e., a single point of the phase space $C(I)$ ) and $[a, b]$ (equivalently $J$ ) to mean the set $\{x \in I \mid a \leq x \leq b\} \subseteq I$. In addition, the pointerval $[a, a]_{c}$, is called a degenerate pointerval and, for ease, is denoted by $a_{c}$. Alternatively, the phrase nondegenerate pointerval will be applied for those pointervals that have distinct extremities in $I$, i.e., $a \neq b$. The phrase "single point" will be used to refer to a single element of $I$.

Secondly, we will give a specific interpretation of the phase space $C(I)$ since it provides us with a very useful tool in studying the induced dynamics of $C(I)$ (see [5], Example 5.1). Precisely, we will build a homeomorphism between $C(I)$ and the two dimensional space $I_{y \geq x}^{2}=$ $=\left\{(x, y) \in I^{2} \mid y \geq x\right\}$, that is, the subset of those points of the square $I^{2}$ that are located on and above the diagonal of $I^{2}$ (the blue area in Fig. 1), so that one can interpret any pointerval of $C(I)$ as an ordinary point in $I_{y \geq x}^{2}$. So, suppose that $[a, b]_{c} \in C(I)$ is an arbitrary pointerval. The following homeomorphism is what we are looking for: $[a, b]_{c} \leftrightarrow(a, b)$, where $(a, b)$ is a point in $I_{y \geq x}^{2}$, whose first and second coordinates are $a$ and $b$ respectively. Therefore, any pointerval in $C(I)$ is uniquely related to a point in $I_{y \geq x}^{2}$ and conversely. For example, if $a$ is any point in $I$, then the pointerval $a_{c} \in C(I)$ is related to a point on the lower vertex of the triangle $I_{y \geq x}^{2}$ with respect to the mentioned homeomorphism. Conversely, any point of the lower vertex of $I_{y \geq x}^{2}$ is related to a degenerate pointerval of $C(I)$. In the remainder, the subset of all degenerate pointervals of $C(I)$ will be denoted by $\mathcal{L}$, that is, $\mathcal{L}=\left\{[a, a]_{c} \in C(I) \mid a \in I\right\}$. Obviously, the set $\mathcal{L}$ is invariant under any induced map $\mathcal{F}$ on $C(I)$. 


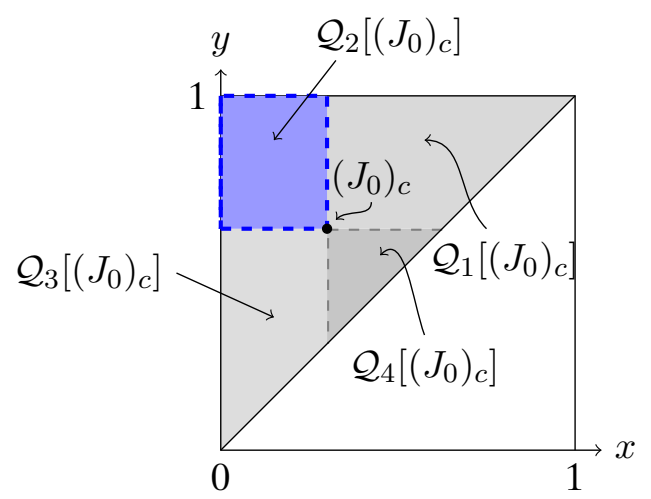

Fig. 2. $\mathcal{Q}_{1}\left[\left(J_{0}\right)_{c}\right], \mathcal{Q}_{2}\left[\left(J_{0}\right)_{c}\right], \mathcal{Q}_{3}\left[\left(J_{0}\right)_{c}\right]$ and $\mathcal{Q}_{4}\left[\left(J_{0}\right)_{c}\right]$.

Here, it is worth mentioning the following point. We are completely aware that the terms such as "intersection", "union", "disjointness", "inclusion" and etc. are naturally being used for expressing relations among sets. Since according to the definition, a pointerval is a single element of the phase space $C(I)$, it does not make much sense to use the above mentioned terms regarding the pointervals. Despite this fact and in order to avoid tediousness of writing extra words, we will sometimes apply such terms for pointervals. We would like the reader to remember that, in such cases, we use these terms for pointervals having in mind as if we regard these pointervals as ordinary subintervals of $I$.

Let $\left(J_{0}\right)_{c}$ be a pointerval in $C(I)$. Then, we define (see Fig. 2)

$\mathcal{Q}_{1}\left[\left(J_{0}\right)_{c}\right]=\left\{J_{c} \in C(I) \mid \min J_{0}<\min J\right.$ and $\left.\max J_{0}<\max J\right\}$;

$\mathcal{Q}_{2}\left[\left(J_{0}\right)_{c}\right]=\left\{J_{c} \in C(I) \mid J_{0} \subseteq J\right\}$

$\mathcal{Q}_{3}\left[\left(J_{0}\right)_{c}\right]=\left\{J_{c} \in C(I) \mid \min J_{0}>\min J\right.$ and $\left.\max J_{0}>\max J\right\}$;

$\mathcal{Q}_{4}\left[\left(J_{0}\right)_{c}\right]=\left\{J_{c} \in C(I) \mid J_{0} \supseteq J\right\}$.

Obviously, we have that $\mathcal{Q}_{1}\left(J_{c}\right) \cup \mathcal{Q}_{2}\left(J_{c}\right) \cup \mathcal{Q}_{3}\left(J_{c}\right) \cup \mathcal{Q}_{4}\left(J_{c}\right)=C(I)$, for any $J_{c} \in C(I)$. Also, $\mathcal{Q}_{2}\left(J_{c}\right) \cap \mathcal{Q}_{4}\left(J_{c}\right)=\left\{J_{c}\right\}$, while $J_{c}$ belongs to neither of the disjoint sets $\mathcal{Q}_{1}\left(J_{c}\right)$ and $\mathcal{Q}_{3}\left(J_{c}\right)$.

Let $\mathcal{N}$ be a set of pointervals, i.e., $\mathcal{N} \subseteq C(I)$. Then, $\mathcal{N}^{\cup}=\bigcup_{J_{c} \in \mathcal{N}} J$ and $\mathcal{N}^{\cap}=\bigcap_{J_{c} \in \mathcal{N}} J$. Obviously, $\mathcal{N}^{\cup}$ and $\mathcal{N}^{\cap}$ are subsets of $I$. If $\mathcal{N}$ is strongly $\mathcal{F}$-invariant, then $\mathcal{N}^{\cup}$ and $\mathcal{N}^{\cap}$ are strongly $f$-invariant (although, $\mathcal{N}^{\cap}$ may happen to be empty). The set $\mathcal{N}=\left\{\left(J_{i}\right)_{c}: i=\right.$ $=1, \ldots, n ; n \in \mathbb{N}\}$ is said to be a cycle under the action of $\mathcal{F}$ (or simply $\mathcal{F}$-cycle), if pointervals $\left(J_{1}\right)_{c},\left(J_{2}\right)_{c}, \ldots,\left(J_{n}\right)_{c}$ produce a cycle under $\mathcal{F}$, that is, $\mathcal{F}\left[\left(J_{i}\right)_{c}\right]=\left(J_{i+1}\right)_{c}$, for all $i=$ $=1,2, \ldots, n-1$, and $\mathcal{F}\left[\left(J_{n}\right)_{c}\right]=\left(J_{1}\right)_{c}$. By $\operatorname{card}(\mathcal{N})$ we denote the cardinality of the set $\mathcal{N}$.

Let $\mathcal{F}: C(I) \rightarrow C(I)$ be a continuous induced map. Then, we call a pointerval $J_{c} \in C(I)$ an eventually periodic pointerval (of period $p \geq 1$ ) under $\mathcal{F}$ if there exists a number $m \geq 0$ such that $\mathcal{F}^{m+p}\left(J_{c}\right)=\mathcal{F}^{m}\left(J_{c}\right)$. In the case where $p=1$ we call $J_{c}$ an eventually fixed pointerval.

Let $\mathcal{S}=\left(\left(J_{i}\right)_{c}\right)_{i=1}^{\infty}$ be a sequence of pointervals, where $J_{i}=\left[a_{i}, b_{i}\right] \subseteq I$. Then, the sequence $\mathcal{S}$ is said to be monotone if the sequences $\left(a_{i}\right)_{i=0}^{\infty}$ and $\left(b_{i}\right)_{i=0}^{\infty}$ are both monotone in $I$. The sequence $\mathcal{S}$ is called bimonotone if both sequences $\mathcal{S}_{1}=\left(\left(J_{2 k-1}\right)_{c}\right)_{k=1}^{\infty}$ and $\mathcal{S}_{2}=$ $=\left(\left(J_{2 k}\right)_{c}\right)_{k=1}^{\infty}$ are monotone. Note that $\mathcal{S}_{1}$ and $\mathcal{S}_{2}$ can converge to different pointervals. So, a 
monotone sequence is necessarily bimonotone, whilst the converse statement is not true (even if $\mathcal{S}_{1}$ and $\mathcal{S}_{2}$ converge to the same pointerval. The monotone sequence $\mathcal{S}$ is called increasing if $\left(J_{i+1}\right)_{c} \in \mathcal{Q}_{2}\left[\left(J_{i}\right)_{c}\right]$ (or equivalently, $\left.J_{i} \subseteq J_{i+1}\right)$, for any $i \geq 1$. Similarly, the monotone sequence $\mathcal{S}$ is called decreasing if $\left(J_{i+1}\right)_{c} \in \mathcal{Q}_{4}\left[\left(J_{i}\right)_{c}\right]$ (equivalently, $J_{i} \supseteq J_{i+1}$ ), for any $i \geq 1$.

3. Existence of the FPP for subsets of $C(I)$. In this section, we will examine some particular subsets of $C(I)$ to find out which of them have the FPP. In spite of the fact that connected subsets of $C(I)$ are seeming the most natural ones to be questioned, we will leave them until the last part of this section. In the first stages, we begin with some of the simpler subsets that are not necessarily connected. Namely, we are going to consider finite subsets of $C(I)$ first, and then we will develop the discussion for infinite subsets as well. Recall that for some of certain finite subsets of the phase space $I$, it is extremely trivial to verify that the FPP does not hold. For example, let $A \subset I$ be a cycle of length $n(\geq 2)$ under a continuous map $f: I \rightarrow I$. Then, obviously it does not have any fixed point. The same thing is true for finite subsets of $C(I)$. However, imposing some additional conditions to a finite set of pointervals can lead to the existence of the FPP.

Before moving forward, let us state a few lemmas that are going to help us in the upcoming discussion. Note that, for technical reasons, we have to frequently switch from the space $C(I)$ to $I$ and conversely. That is why we often use $\mathcal{F}$ and $f$ almost simultaneously in the same context when we move from one space to the other. Although, in some cases one can apply either of them without changing the concept.

\subsection{Auxiliary lemmas.}

Lemma 1. Let $\left(J_{1}\right)_{c} \in C(I)$ be a nondegenerate pointerval and $\mathcal{F}: C(I) \rightarrow C(I)$ be a continuous induced map. Also, let $\left(J_{i}\right)_{c}=\mathcal{F}^{i-1}\left[\left(J_{1}\right)_{c}\right]$, for $i=1,2, \ldots$ If there is a number $m \geq 2$ such that $\left(J_{m}\right)_{c} \in\left\{\mathcal{Q}_{2}\left[\left(J_{1}\right)_{c}\right] \cup \mathcal{Q}_{4}\left[\left(J_{1}\right)_{c}\right]\right\} \backslash\left\{\left(J_{1}\right)_{c}\right\}$, then there cannot exist any number $k \geq 1$ such that $\mathcal{F}^{k}\left[\left(J_{m}\right)_{c}\right]=\left(J_{1}\right)_{c}$.

Proof. Obviously, if $\left(J_{2}\right)_{c} \in\left\{\mathcal{Q}_{2}\left[\left(J_{1}\right)_{c}\right] \cup \mathcal{Q}_{4}\left[\left(J_{1}\right)_{c}\right]\right\} \backslash\left\{\left(J_{1}\right)_{c}\right\}$ (or equivalently $J_{2} \subset J_{1}$ or $\left.J_{2} \supset J_{1}\right)$, then for any $k \geq 2$ we have that $\left(J_{k}\right)_{c} \in\left\{\mathcal{Q}_{2}\left[\left(J_{1}\right)_{c}\right] \cup \mathcal{Q}_{4}\left[\left(J_{1}\right)_{c}\right]\right\} \backslash\left\{\left(J_{1}\right)_{c}\right\}$ and hence $\left(J_{k}\right)_{c} \neq\left(J_{1}\right)_{c}$.

Now, assume that $m \geq 3$ be a number such that $\left(J_{m}\right)_{c} \in\left\{\mathcal{Q}_{2}\left[\left(J_{1}\right)_{c}\right] \cup \mathcal{Q}_{4}\left[\left(J_{1}\right)_{c}\right]\right\} \backslash\left\{\left(J_{1}\right)_{c}\right\}$ and $\left(J_{i}\right)_{c} \in C(I) \backslash\left\{\mathcal{Q}_{2}\left[\left(J_{1}\right)_{c}\right] \cup \mathcal{Q}_{4}\left[\left(J_{1}\right)_{c}\right]\right\}$, for $i=2,3, \ldots, m-1$. This implies that $\left(J_{m+k}\right)_{c} \in$ $\in\left\{\mathcal{Q}_{2}\left[\left(J_{k+1}\right)_{c}\right] \cup \mathcal{Q}_{4}\left[\left(J_{k+1}\right)_{c}\right] \cup \mathcal{Q}_{2}\left[\left(J_{1}\right)_{c}\right] \cup \mathcal{Q}_{4}\left[\left(J_{1}\right)_{c}\right]\right\} \backslash\left\{\left(J_{1}\right)_{c}\right\}$, for any $k \geq 1$, and this completes the proof.

Lemma 2. Let $\left(J_{i}\right)_{c}, i=1,2,3$, be three nondegenerate pointervals in $C(I)$ such that $\left(J_{1}\right)_{c} \neq$ $\neq\left(J_{2}\right)_{c},\left(J_{1}\right)_{c} \neq\left(J_{3}\right)_{c}$ and $J_{1} \cap J_{2} \cap J_{3} \neq \varnothing$. Also, let $\mathcal{F}: C(I) \rightarrow C(I)$ be a continuous induced map such that $\mathcal{F}\left[\left(J_{i}\right)_{c}\right]=\left(J_{i+1}\right)_{c}$, for $i=1,2$. Then, there does not exist any number $k \geq 1$, for which holds $\mathcal{F}^{k}\left[\left(J_{3}\right)_{c}\right]=\left(J_{1}\right)_{c}$.

Proof. Denote $\left(J_{i}\right)_{c}=\mathcal{F}^{i-1}\left[\left(J_{1}\right)_{c}\right]$, for all $i \geq 1$. Let us consider all the possibilities that can happen step by step.

The easiest case is when for any $k \geq 2$ we have that $\left(J_{k}\right)_{c}$ is fixed. If this is the case, then obviously there is nothing to prove. Hence, from this moment on we assume that this is not the case.

First, if $\left(J_{2}\right)_{c} \in\left\{\mathcal{Q}_{2}\left[\left(J_{1}\right)_{c}\right] \cup \mathcal{Q}_{4}\left[\left(J_{1}\right)_{c}\right]\right\} \backslash\left\{\left(J_{1}\right)_{c}\right\}$, then obviously the desired statement holds and there is nothing left to prove. 
So, assume that $\left(J_{2}\right)_{c} \in \mathcal{Q}_{1}\left[\left(J_{1}\right)_{c}\right]$ or $\left(J_{2}\right)_{c} \in \mathcal{Q}_{3}\left[\left(J_{1}\right)_{c}\right]$. We discuss the first case thoroughly, but will leave the second one to the reader as they are analogous.

Hence, suppose that $\left(J_{2}\right)_{c} \in \mathcal{Q}_{1}\left[\left(J_{1}\right)_{c}\right]$. At this moment again there can occur different possibilities. If $\left(J_{3}\right)_{c} \in \mathcal{Q}_{2}\left[\left(J_{2}\right)_{c}\right] \cup \mathcal{Q}_{4}\left[\left(J_{2}\right)_{c}\right]$, then we are done. But if $\left(J_{3}\right)_{c} \in\left\{\mathcal{Q}_{2}\left[\left(J_{1}\right)_{c}\right] \cup\right.$ $\left.\cup \mathcal{Q}_{4}\left[\left(J_{1}\right)_{c}\right]\right\} \backslash\left\{\left(J_{1}\right)_{c}\right\}$, then according to Lemma $1,\left(J_{3}\right)_{c}$ can never return to $\left(J_{1}\right)_{c}$ under $\mathcal{F}$ iterations. In fact, Lemma 1 implies that if for any $i \geq 2$ we have that $\left(J_{i}\right)_{c} \in \mathcal{Q}_{2}\left[\left(J_{1}\right)_{c}\right] \cup$ $\cup \mathcal{Q}_{4}\left[\left(J_{1}\right)_{c}\right] \cup \mathcal{Q}_{2}\left[\left(J_{2}\right)_{c}\right] \cup \mathcal{Q}_{4}\left[\left(J_{2}\right)_{c}\right] \cup \ldots \cup \mathcal{Q}_{2}\left[\left(J_{i-1}\right)_{c}\right] \cup \mathcal{Q}_{4}\left[\left(J_{i-1}\right)_{c}\right]$, then $\left(J_{3}\right)_{c}$ cannot come back to $\left(J_{1}\right)_{c}$ under $\mathcal{F}$. Therefore, we assume that this never happens. Assume that $\left(J_{3}\right)_{c} \in \mathcal{Q}_{1}\left[\left(J_{2}\right)_{c}\right]$. More precisely, we have that $\left(J_{2}\right)_{c} \in \mathcal{Q}_{1}\left[\left(J_{1}\right)_{c}\right]$ and $\left(J_{3}\right)_{c} \in \mathcal{Q}_{1}\left[\left(J_{2}\right)_{c}\right]$ (see Fig. 3(a)). In this case, obviously, holds the inclusion $J_{2} \subset J_{1} \cup J_{3}$. But the last inclusion implies that $J_{3} \subset J_{2} \cup J_{4}$, which, in turn, means that $\left(J_{i}\right)_{c} \neq\left(J_{1}\right)_{c}$, for any $i \geq 4$.

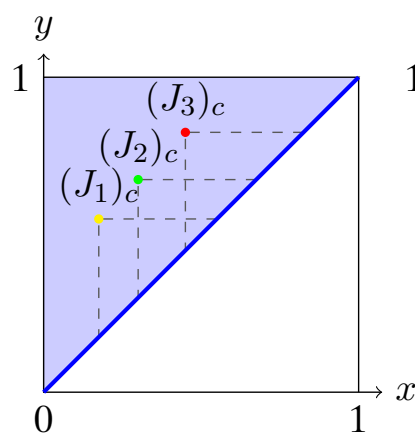

(a)

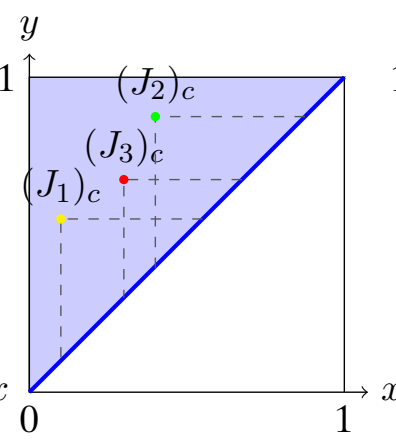

(b)

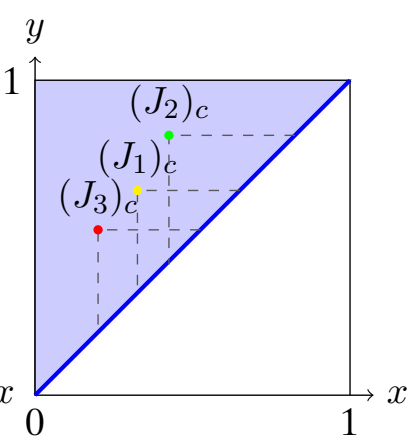

(c)

Fig. 3. Proof of Lemma 2.

Now, suppose that $\left(J_{2}\right)_{c} \in \mathcal{Q}_{1}\left[\left(J_{1}\right)_{c}\right]$ and $\left(J_{3}\right)_{c} \in \mathcal{Q}_{1}\left[\left(J_{1}\right)_{c}\right] \cap \mathcal{Q}_{3}\left[\left(J_{2}\right)_{c}\right]$ (see Fig. 3(b)). One can easily check that if this is the case, then holds $J_{3} \subset J_{1} \cup J_{2}$. But, this implies that $J_{i+2} \subset$ $\subset J_{i} \cup J_{i+1}$, for all $i \geq 1$. In other words, for any $i \geq 4$, we have that $\min J_{1}<\min J_{3}<\min J_{i}$, i.e., $\left(J_{i}\right)_{c} \neq\left(J_{1}\right)_{c}$. Next, assume that $\left(J_{2}\right)_{c} \in \mathcal{Q}_{1}\left[\left(J_{1}\right)_{c}\right]$ and $\left(J_{3}\right)_{c} \in \mathcal{Q}_{3}\left[\left(J_{1}\right)_{c}\right]$ (see Fig. 3(c)). It is easy to see that we have $J_{1} \subset J_{2} \cup J_{3}$. This implies that $J_{i} \subset J_{i+1} \cup J_{i+2}$, for all $i \geq 1$. This means that if $i$ is an even number, then we have that $\max J_{1}<\max J_{2}<\max J_{i}$, and if $i$ is an odd number, then $\min J_{i}<\min J_{3}<\min J_{1}$. So, obviously we have that $\left(J_{i}\right)_{c} \neq\left(J_{1}\right)_{c}$, for any $i \geq 4$. Therefore, we discussed all the possible variants for the case where $\left(J_{2}\right)_{c} \in \mathcal{Q}_{1}\left[\left(J_{1}\right)_{c}\right]$ and, as we mentioned before, the second case $\left(J_{2}\right)_{c} \in \mathcal{Q}_{3}\left[\left(J_{1}\right)_{c}\right]$ is left to the reader.

Lemma 2 is proved.

Lemma 3. Let $\mathcal{N}=\left\{\left(J_{i}\right)_{c}: i=1, \ldots, n ; n \in \mathbb{N}\right\} \subset C(I)$ be a set of nondegenerate pointervals such that $\mathcal{N}^{\cup}$ is a connected subset of I. If $\mathcal{N}$ is a cycle with respect to a continuous induced map $\mathcal{F}: C(I) \rightarrow C(I)$, then $\operatorname{card}(\mathcal{N}) \leq 2$.

Proof. By way of contradiction, assume that $n=\operatorname{card}(\mathcal{N}) \geq 3$. It is clear that if $\mathcal{N}$ is a cycle with respect to $\mathcal{F}$, then the set $\mathcal{N}^{\cup}$ is strongly invariant under $f$. This implies that there is a single point $x \in \mathcal{N}^{\cup}$, for which holds $f(x)=x$. Hence, there is at least one pointerval $\left(J_{i}\right)_{c} \in$ $\in \mathcal{N}, i \in\{1, \ldots, n\}$ such that $x \in J_{i}$. If this $\left(J_{i}\right)_{c}$ is the only pointerval of $\mathcal{N}$ that contains $x$, then $\mathcal{F}\left[\left(J_{i}\right)_{c}\right]=\left(J_{i}\right)_{c}$ since $x$ is an $f$-fixed point and this is obviously a contradiction as we assumed that $\mathcal{N}$ is a cycle with at least three pointervals. So, there definitely exists another 
pointerval $\left(J_{j}\right)_{c} \in \mathcal{N}, j \in\{1, \ldots, n\}, i \neq j$, that has the point $x$ inside. Again and similarly as above, we have that $\mathcal{F}\left[\left(J_{j}\right)_{c}\right] \neq\left(J_{j}\right)_{c}$. In addition, $\left.\mathcal{F}\left[J_{j}\right)_{c}\right] \neq\left(J_{i}\right)_{c}$ as they cannot produce a cycle of period two. In the same way, one can conclude that $x$ belongs to the all pointervals of $\mathcal{N}$. In particular, we have that $J_{1} \cap J_{2} \cap J_{3} \neq \varnothing$ and according to Lemma 2 there does not exist any number $k \geq 1$ such that $\mathcal{F}^{k}\left[\left(J_{3}\right)_{c}\right]=\left(J_{1}\right)_{c}$. Therefore, the assumption $n=\operatorname{card}(\mathcal{N}) \geq 3$ we made by contradiction is not true. On the other hand, it is primitive to check that if $\operatorname{card}(\mathcal{N}) \leq$ 2 , then the set $\mathcal{N}$ can perfectly be a cycle.

Lemma 3 is proved.

Lemma 4. Let $\mathcal{N}_{i}=\left\{\left(J_{1}^{i}\right)_{c},\left(J_{2}^{i}\right)_{c}, \ldots,\left(J_{n_{i}}^{i}\right)_{c}\right\} \subset C(I), 1 \leq i \leq n, n_{i} \in \mathbb{N}$, be sets of nondegenerate pointervals such that $\mathcal{N}_{i}^{\cup}$, for any $i \in\{1, \ldots, n\}$, is a connected subset of $I$. Also, let for any $i, j \in\{1, \ldots, n\}, i \neq j$, holds $\mathcal{N}_{i}^{\cup} \cap \mathcal{N}_{j}^{\cup}=\varnothing$. If $\mathcal{N}=\bigcup_{i=1}^{n} \mathcal{N}_{i}$ is a cycle under a continuous induced map $\mathcal{F}: C(I) \rightarrow C(I)$, then $\operatorname{card}\left(\mathcal{N}_{i}\right) \leq 2$, for any $i \in\{1, \ldots, n\}$.

Proof. It is not difficult to verify that if $\mathcal{N}$ is a cycle under $\mathcal{F}$, then all the $\mathcal{N}_{i}$ 's have the same cardinality and, in addition, each $\mathcal{N}_{i}$ is a cycle with respect to $\mathcal{G}=\mathcal{F}^{n}$. Hence, according to Lemma 3, we have that $\operatorname{card}\left(\mathcal{N}_{i}\right) \leq 2$, for any $i \in\{1, \ldots, n\}$.

3.2. Finite subsets of $C(I)$. Now, let us go back to the question mentioned at the beginning of Section 3. As it was mentioned there, by adding some more conditions to a finite set of $C(I)$ we may obtain the FPP. By adding these conditions the range of finite sets we are going to discuss will narrow. Answering the question relating the existence of FPP for all finite subsets of $C(I)$ needs further investigation. So, let us assume that $\mathcal{N} \subset C(I)$ is a nonempty, finite and strongly $\mathcal{F}$-invariant set such that $\mathcal{N} \cap \neq \varnothing$. The question is then whether $\mathcal{N}$ always contains a fixed, under $\mathcal{F}$, pointerval or not. First, it is clear that each element of a finite and strongly invariant set (no matter which phase space we are in) is periodic of period $p(\geq 1)$. This fact together with the following lemma are the key factors to answer the question.

Lemma 5. Let $\mathcal{N}=\left\{\left(J_{i}\right)_{c}: i=1, \ldots, n ; n \in \mathbb{N}\right\} \subset C(I)$ be a nonempty set such that $\mathcal{N}^{\cap} \neq \varnothing$. Also, let $\mathcal{F}: C(I) \rightarrow C(I)$ be a continuous induced map.

(a) If $\mathcal{N}$ is $\mathcal{F}$-invariant, then each pointerval of $\mathcal{N}$ is eventually fixed or eventually periodic of period $p=2$ with respect to $\mathcal{F}$.

(b) If $\mathcal{N}$ is strongly $\mathcal{F}$-invariant, then each pointerval of $\mathcal{N}$ is fixed or periodic of period $p=2$ with respect to $\mathcal{F}$.

Proof. Suppose that $\left(J_{i}\right)_{c}$ is an arbitrary pointerval in $\mathcal{N}$. As $\mathcal{N}$ is invariant with respect to $\mathcal{F}$ and $\mathcal{N}^{\cap} \neq \varnothing$, Lemma 3 implies that if $\left(J_{i}\right)_{c}$ is a periodic pointerval of period $p$, then $p \leq 2$. Now, let us see what will happen if $\left(J_{i}\right)_{c}$ is not periodic at all. If $\left(J_{i}\right)_{c}$ never returns to itself under $\mathcal{F}$-iterations, and having in mind that $\mathcal{N}$ is $\mathcal{F}$-invariant, then there definitely exists a number $m \geq 1$ such that $\mathcal{F}^{m}\left[\left(J_{i}\right)_{c}\right]$ is fixed or of period two. Hence, until now we have proved that if $\mathcal{N}$ is only invariant, then any arbitrary pointerval $\left(J_{i}\right)_{c}$ of $\mathcal{N}$ is eventually fixed or eventually periodic of period two. But if $\mathcal{N}$ is strongly invariant, then any pointerval $\left(J_{i}\right)_{c} \in \mathcal{N}$ has to be periodic. This together with what we have just shown above lead us to the fact that $\left(J_{i}\right)_{c}$ is either fixed or of period 2.

Lemma 5 is proved.

Now, and according to Lemma 5, it is casy to see that the following theorem is true.

Theorem 1. Let $\mathcal{N}=\left\{\left(J_{i}\right)_{c}: i=1, \ldots, n ; n \in \mathbb{N}\right\} \subset C(I)$ be a nonempty set such that $\mathcal{N}^{\cap} \neq \varnothing$. Also, let $\mathcal{F}: C(I) \rightarrow C(I)$ be a continuous induced map. 
(a) If $\mathcal{N}$ is $\mathcal{F}$-invariant, then it contains a periodic pointerval of period $p \leq 2$ with respect to $\mathcal{F}$;

(b) If $\mathcal{N}$ is strongly $\mathcal{F}$-invariant and $\operatorname{card}(\mathcal{N})$ is odd, then it necessarily contains a fixed pointerval with respect to $\mathcal{F}$.

3.3. Infinite subsets of $C(I)$. Now in this part of the paper, we would like to discuss the question regarding the FPP for infinite subsets of $C(I)$. As in the previous part, we will add similar conditions to infinite sets of $C(I)$ and will focus only on this sort of sets.

More precisely, let $\mathcal{O} \subset C(I)$ be a nonempty, closed and infinite set such that $\mathcal{O}^{\cap} \neq \varnothing$. Also, let $\mathcal{O}$ be an $\mathcal{F}$-invariant set. Lemma 6 helps us characterize the trajectories of pointervals that belong to $\mathcal{O}$.

Lemma 6. Let $\left(J_{i}\right)_{c}, i=1,2,3$, be three pointervals in $C(I)$ such that $J_{1} \cap J_{2} \cap J_{3} \neq \varnothing$. Also, let $\mathcal{F}: C(I) \rightarrow C(I)$ be a continuous induced map such that $\mathcal{F}\left[\left(J_{i}\right)_{c}\right]=\left(J_{i+1}\right)_{c}$, for $i=1,2$. Then, there exists a number $m \geq 1$ such that the sequence $\left(\left(J_{i}\right)_{c}\right)_{i=m}^{\infty}$ is bimonotonic.

Proof. Denote $\left(J_{i}\right)_{c}=\mathcal{F}^{i-1}\left[\left(J_{1}\right)_{c}\right]$, for all $i \geq 1$. Suppose that $\left(J_{1}\right)_{c}$ is any pointerval in $\mathcal{O}$. We know that $\mathcal{O}$ is $\mathcal{F}$-invariant and, in addition, we have that $\mathcal{O}^{\cap} \neq \varnothing$. So, according to Lemma 3 , if $\left(J_{1}\right)_{c}$ has a finite trajectory, then it has to be an eventually fixed or eventually periodic of period $p=2$.

Now, assume that $\left(J_{1}\right)_{c} \in \mathcal{O}$ is any pointerval that has an infinite trajectory. First, if there is a number $m \geq 1$ such that $\left(J_{m+1}\right)_{c} \in \mathcal{Q}_{2}\left[\left(J_{m}\right)_{c}\right] \cup \mathcal{Q}_{4}\left[\left(J_{m}\right)_{c}\right]$, then obviously the sequence $\left(\left(J_{i}\right)_{c}\right)_{i=m}^{\infty}$ is either increasing or decreasing and, hence, is bimonotone. So, assume that this does not happen. Therefore, one of the following can occur:

$\left(J_{2}\right)_{c} \in \mathcal{Q}_{1}\left[\left(J_{1}\right)_{c}\right]$ or $\left(J_{2}\right)_{c} \in \mathcal{Q}_{3}\left[\left(J_{1}\right)_{c}\right]$. As the argumentation for both of these cases are analogous, similarly as in Lemma 2, we only discuss the first one and leave the second one to the reader.

So, suppose that $\left(J_{2}\right)_{c} \in \mathcal{Q}_{1}\left[\left(J_{1}\right)_{c}\right]$. If $\left(J_{3}\right)_{c} \in \mathcal{Q}_{2}\left[\left(J_{1}\right)_{c}\right] \backslash\left\{\left(J_{1}\right)_{c}\right\}$ or $\left(J_{3}\right)_{c} \in \mathcal{Q}_{4}\left[\left(J_{1}\right)_{c}\right] \backslash$ $\left\{\left(J_{1}\right)_{c}\right\}$, then we have that $\left(J_{i+2}\right)_{c} \in \mathcal{Q}_{2}\left[\left(J_{i}\right)_{c}\right]$ or $\left(J_{i+2}\right)_{c} \in \mathcal{Q}_{4}\left[\left(J_{i}\right)_{c}\right]$, for all $i \geq 1$, respectively. And this clearly means that the sequence $\left(\left(J_{i}\right)_{c}\right)_{i=1}^{\infty}$ is bimonotone. As a matter of fact, this can happen any time, that is, it is generally possible that there is a number $m \geq 1$ such that $\left(J_{m+2}\right)_{c} \in \mathcal{Q}_{2}\left[\left(J_{m}\right)_{c}\right] \backslash\left\{\left(J_{m}\right)_{c}\right\}$ or $\left(J_{m+2}\right)_{c} \in \mathcal{Q}_{4}\left[\left(J_{m}\right)_{c}\right] \backslash\left\{\left(J_{m}\right)_{c}\right\}$, and in both cases we have that the sequence $\left(\left(J_{i}\right)_{c}\right)_{i=m}^{\infty}$ is bimonotone.

Now, suppose that $\left(J_{2}\right)_{c} \in \mathcal{Q}_{1}\left[\left(J_{1}\right)_{c}\right]$ and $\left(J_{3}\right)_{c} \in \mathcal{Q}_{1}\left[\left(J_{2}\right)_{c}\right]$ (Fig. 3(a)). One can easily show that in this case (and according to the assumption $\left(J_{i+1}\right)_{c} \notin \mathcal{Q}_{2}\left[\left(J_{i}\right)_{c}\right] \cup \mathcal{Q}_{4}\left[\left(J_{i}\right)_{c}\right]$, for any $i \geq 1$, that we made at the beginning of the proof), the only possible scenario that can take place is that $\left(J_{i+1}\right)_{c} \in \mathcal{Q}_{1}\left[\left(J_{i}\right)_{c}\right]$, for all $i \geq 1$. And this means that the sequence $\left(\left(J_{i}\right)_{c}\right)_{i=1}^{\infty}$ is a monotone and hence bimonotone sequence.

The next possibility is that $\left(J_{2}\right)_{c} \in \mathcal{Q}_{1}\left[\left(J_{1}\right)_{c}\right]$ and $\left(J_{3}\right)_{c} \in \mathcal{Q}_{1}\left[\left(J_{1}\right)_{c}\right] \cap \mathcal{Q}_{3}\left[\left(J_{2}\right)_{c}\right]$ (Fig. 3(b)). Also, we have that $\left(J_{i+2}\right)_{c} \notin \mathcal{Q}_{2}\left[\left(J_{i}\right)_{c}\right] \cup \mathcal{Q}_{4}\left[\left(J_{i}\right)_{c}\right] \cup \mathcal{Q}_{2}\left[\left(J_{i+1}\right)_{c}\right] \cup \mathcal{Q}_{4}\left[\left(J_{i+1}\right)_{c}\right]$, for any $i \geq 1$. If this is the case, then we have that $J_{3} \subset J_{1} \cup J_{2}$, which implies that $J_{i+2} \subset J_{i} \cup J_{i+1}$. And this means that for any odd number $i \geq 1$, we have that $\left(J_{i+2}\right)_{c} \in \mathcal{Q}_{1}\left[\left(J_{i}\right)_{c}\right]$ and for any even number $i \geq 2$ we have that $\left(J_{i+2}\right)_{c} \in \mathcal{Q}_{3}\left[\left(J_{i}\right)_{c}\right]$. And this is exactly what we were looking for. 
The last possibility is when we have that $\left(J_{2}\right)_{c} \in \mathcal{Q}_{1}\left[\left(J_{1}\right)_{c}\right]$ and $\left(J_{3}\right)_{c} \in \mathcal{Q}_{3}\left[\left(J_{1}\right)_{c}\right]$ (Fig. 3(c)). Exactly the same as in the previous cases, we assume that $\left(J_{i+2}\right)_{c} \notin \mathcal{Q}_{2}\left[\left(J_{i}\right)_{c}\right] \cup \mathcal{Q}_{4}\left[\left(J_{i}\right)_{c}\right] \cup$ $\cup \mathcal{Q}_{2}\left[\left(J_{i+1}\right)_{c}\right] \cup \mathcal{Q}_{4}\left[\left(J_{i+1}\right)_{c}\right]$, for any $i \geq 1$. Clearly, we have that $J_{1} \subset J_{2} \cup J_{3}$, which in turn implies that $J_{i} \subset J_{i+1} \cup J_{i+2}$, for all $i \geq 1$. Hence, for any odd number $i \geq 1$, we have that $\left(J_{i+2}\right)_{c} \in \mathcal{Q}_{3}\left[\left(J_{i}\right)_{c}\right]$, and for any even number $i \geq 2$ we have that $\left(J_{i+2}\right)_{c} \in \mathcal{Q}_{1}\left[\left(J_{i}\right)_{c}\right]$, i.e., the sequence $\left(\left(J_{i}\right)_{c}\right)_{i=1}^{\infty}$ is bimonotone.

Lemma 6 is proved.

In fact, the next corollary immediately follows from Lemma 6.

Corollary 1. Let $\mathcal{O} \subset C(I)$ be a nonempty, closed and infinite set such that $\mathcal{O}^{\cap} \neq \varnothing$. Also, let $\mathcal{F}: C(I) \rightarrow C(I)$ be a continuous induced map. If $\mathcal{O}$ is invariant with respect to $\mathcal{F}$, then for any pointerval $J_{c} \in \mathcal{O}$, holds either of the following:

(a) the pointerval $J_{c}$ is eventually periodic of period $p \leq 2$;

(b) the trajectory of $J_{c}$ is infinite and there is a number $m \geq 1$ such that the sequence $\left(\mathcal{F}^{i}\left(J_{c}\right)\right)_{i=m}^{\infty}$ is bimonotone.

Note that if a sequence $\left(\left(J_{i}\right)_{c}\right)_{i=1}^{\infty}$ is bimonotone, then the subsequences $\left(\left(J_{2 k-1}\right)_{c}\right)_{k=1}^{\infty}$ and $\left(\left(J_{2 k}\right)_{c}\right)_{k=1}^{\infty}$ not necessarily converge to the same pointerval. And as it is possible that these subsequences converge to two different pointervals, hence the described set $\mathcal{O}$ in Corollary 1 does not necessarily contain an $\mathcal{F}$-fixed pointerval inside. On the other hand, it definitely contains a pointerval of period two. Therefore, the next theorem holds:

Theorem 2. Let $\mathcal{O} \subset C(I)$ be a nonempty, closed and infinite set such that $\mathcal{O}^{\cap} \neq \varnothing$. Also, let $\mathcal{F}: C(I) \rightarrow C(I)$ be a continuous induced map. If $\mathcal{O}$ is invariant with respect to $\mathcal{F}$, then there exists a pointerval $J_{c} \in \mathcal{O}$ with $\mathcal{F}^{2}\left(J_{c}\right)=J_{c}$.

3.4. Subcontinuums of $C(I)$. In the remainder, we prove that any nonempty subcontinuum of $C(I)$ has the FPP.

Theorem 3. Let $\mathcal{C}$ be a nonempty continuum of $C(I)$ and $\mathcal{F}: C(I) \rightarrow C(I)$ be a continuous induced map. If $\mathcal{C}$ is invariant with respect to $\mathcal{F}$, then $\mathcal{C}$ has the FPP.

Proof. Suppose that $\left(J_{0}\right)_{c}$ is an arbitrary pointerval in $\mathcal{C}$. It is obvious that holds at least one of the following inclusions:

(a) $\mathcal{F}\left[\left(J_{0}\right)_{c}\right] \in \mathcal{Q}_{1}\left[\left(J_{0}\right)_{c}\right] \cap \mathcal{C}$,

(b) $\mathcal{F}\left[\left(J_{0}\right)_{c}\right] \in \mathcal{Q}_{2}\left[\left(J_{0}\right)_{c}\right] \cap \mathcal{C}$,

(c) $\mathcal{F}\left[\left(J_{0}\right)_{c}\right] \in \mathcal{Q}_{3}\left[\left(J_{0}\right)_{c}\right] \cap \mathcal{C}$,

(d) $\mathcal{F}\left[\left(J_{0}\right)_{c}\right] \in \mathcal{Q}_{4}\left[\left(J_{0}\right)_{c}\right] \cap \mathcal{C}$.

Note that we said "at least"one the above mentioned items holds, because clearly in general case (b) and (d) can happen simultaneously. But if this is the case, then $\left(J_{0}\right)_{c}$ is a fixed pointerval under $\mathcal{F}$ and there is nothing left to prove. So, suppose that for any pointerval of $\mathcal{C}$ occurs exactly one of the above inclusions. Let $\mathcal{C}_{1}=\left\{J_{c} \in \mathcal{C}: \mathcal{F}\left(J_{c}\right) \in \mathcal{Q}_{1}\left(J_{c}\right)\right\}, \mathcal{C}_{2}=\left\{J_{c} \in \mathcal{C}\right.$ : $\left.\mathcal{F}\left(J_{c}\right) \in \mathcal{Q}_{2}\left(J_{c}\right)\right\}, \mathcal{C}_{3}=\left\{J_{c} \in \mathcal{C}: \mathcal{F}\left(J_{c}\right) \in \mathcal{Q}_{3}\left(J_{c}\right)\right\}$ and $\mathcal{C}_{4}=\left\{J_{c} \in \mathcal{C}: \mathcal{F}\left(J_{c}\right) \in \mathcal{Q}_{4}\left(J_{c}\right)\right\}$. Clearly, we have that $\mathcal{C}=\mathcal{C}_{1} \cup \mathcal{C}_{2} \cup \mathcal{C}_{3} \cup \mathcal{C}_{4}$. First, we show that $\mathcal{C}_{2}$ and $\mathcal{C}_{4}$ cannot be empty simultaneously, that is, $\mathcal{C}_{2} \cup \mathcal{C}_{4} \neq \varnothing$.

Seeking a contradiction, suppose that $\mathcal{C}_{2} \cup \mathcal{C}_{4}=\varnothing$. Consequently, we obtain that $\mathcal{C}=\mathcal{C}_{1} \cup \mathcal{C}_{3}$. But this is a contradiction as the nonempty connected set $\mathcal{C}$ cannot be expressed as a union of two open (with respect to the induced topology on $\mathcal{C}$ ) and pairwise disjoint sets $\mathcal{C}_{1}$ and $\mathcal{C}_{3}$. In 
order to understand why $\mathcal{C}_{1}$ is open, take an arbitrary pointerval $\left(J_{0}\right)_{c}$ in $\mathcal{C}_{1}$. Then, it is not hard to verify that there always exists an $\varepsilon>0$ such that $B_{\varepsilon}\left[\left(J_{0}\right)_{c}\right] \subset \mathcal{C}_{1}$, where $B_{\varepsilon}\left[\left(J_{0}\right)_{c}\right]=\left\{J_{c} \in\right.$ $\left.\in \mathcal{C}: \operatorname{dist}_{\mathrm{H}}\left[J_{c},\left(J_{0}\right)_{c}\right]<\varepsilon\right\}$. This means that $\left(J_{0}\right)_{c}$ is an interior point for $\mathcal{C}_{1}$ and from here implies that $\mathcal{C}_{1}$ is open in $\mathcal{C}$. Similarly, one can prove that $\mathcal{C}_{3}$ is open as well. Until now, we proved that the assumption we made by contradiction is not true and hence we have that $\mathcal{C}_{2} \cup \mathcal{C}_{4} \neq \varnothing$.

Now, we will show that the fact that not both $\mathcal{C}_{2}$ and $\mathcal{C}_{4}$ are empty at the same time guarantees the existence of a fixed pointerval in $\mathcal{C}$. With no loss of generality, assume that $\mathcal{C}_{2} \neq \varnothing$. Also, suppose that $\left(J_{1}\right)_{c}$ be any pointerval in $\mathcal{C}_{2}$. We have that $\mathcal{F}^{i+1}\left[\left(J_{1}\right)_{c}\right] \in \mathcal{Q}_{2}\left[\mathcal{F}^{i}\left[\left(J_{1}\right)_{c}\right]\right]$, for any $i \geq 0$. Hence, $\left(\mathcal{F}^{i}\left[\left(J_{1}\right)_{c}\right]\right)_{i=0}^{\infty}$ is an increasing sequence of pointervals in the bounded set $\mathcal{C}$. So, $\left(\mathcal{F}^{i}\left[\left(J_{1}\right)_{c}\right]\right)_{i=0}^{\infty}$ is convergent to a fixed pointerval in $\mathcal{C}$.

1. Acosta G., Illanes A., Méndez-Lango H. The transitivity of induced maps // Topology and Appl. - 2009. 156, № 5. - P. 1013 - 1033.

2. Akin E. Countable metric spaces and chain transitivity. - Preprint, 2013.

3. Fedorenko V. V. Asymptotic periodicity of the trajectories of an interval // Ukr. Math. J. - 2009. - 61, № 6. P. $854-858$.

4. Fedorenko V. V., Romanenko E. Yu., Sharkovsky A. N. Trajectories of intervals in one-dimensional dynamical systems // J. Difference Equat. and Appl. - 2007. - 13. - P. 821 - 828.

5. Illanes A., Nadler S. B. (Jr.). Hyperspaces fundamentals and recent advances // Monogr. and Textbooks in Pure and Appl. Math. - New York: Marcel Dekker, Inc., 1999. - 216.

6. Kolyada S., Robatian D. On omega-limit sets of triangular induced maps // Real Analysis Exchange. 2013. - 38. - P. 299-316.

7. Kwietniak D., Oprocha P. Topological entropy and chaos for maps induced on hyperspaces // Chaos, Solitons, Fractals. - 2007. - 33, № 1. - P. 76-86.

8. Matviichuk M. On the dynamics of subcontinua of a tree // J. Difference Equat. and Appl. - 2013. - 19, № 2. - P. 223-233.

9. Nadler S. B. (Jr.). Continuum theory: an Introduction // Monogr. and Textbooks in Pure and Appl. Math. New York: Marcel Dekker, 1992. - 158.

10. Schreffler M. Fixed point theorems in topology and geometry // Topology and Appl. - 2009. - 156, № 5. P. $1013-1033$.

Received 11.09.14 